\title{
Simulation Of an Innovative Public Transport System
}

\author{
Philip Kilby $^{\text {a b }} \underline{\text { Matt Robards }}^{\text {a }}$ \\ ${ }^{a}$ Optimisation Research Group, NICTA, Locked Bag 8001 Canberra ACT 2601 \\ ${ }^{\mathrm{b}}$ Australian National University \\ Email: Matthew.Robards@nicta.com.au
}

\begin{abstract}
We report on the use of simulation in the design of in an innovative public transport system. We begin by giving a broad outline of an off-peak public transport system, being designed with the intention of a first implementation replacing weekend services in Canberra, Australia.

The proposed system is based on a small number of carefully selected hub nodes within the city. Hub nodes are linked by high-frequency bus service along trunk routes. A multi-hire taxi system is used to shuttle passengers between their origin or destination stop and the nearest hub.

In this paper we present the results of a simulation of this system. The simulation was run using actual travel data gathered from weekend bus use in Canberra. The simulation was used to test various design options, and helped to shape several aspects of the overall system design.

The main contribution of this paper is to explore the contributions to system design made using insights gathered from the simulation.

For example, contrary to expectation, we saw that a small number of nodes gave the best economic performance. We had expected that a larger bus network would allow the use of taxis to be minimised. However, a smaller number of nodes allows the demand to be concentrated better, allowing more effective use of taxis (higher occupancy).

Another early design feature that was overturned was the requirement that passengers are always shuttled to their closest hub. In some instances it is more efficient - both for the passenger and the taxi - to travel direct to the passenger's destination.

We discuss these and other aspects of the modelling and simulation of the system. We also present preliminary results of the simulations. The results show that for a real demand scenario in Canberra, Australia, the proposed system is able to achieve a one-third reduction in travel time over the current fixed-schedule bus system, without any increase in costs.
\end{abstract}

Keywords: Public Transport, Multimodal, Demand-responsive 


\section{INTRODUCTION}

There exist many mid-sized cities worldwide in which the population is large enough to support a conventional bus system on weekdays, but where off-peak demand is much lower. In such a situation, off-peak services are often infrequent and less convenient. In this paper we investigate aspects of simulating a novel public transport system designed to improve service in off peak times, and aims to be no more expensive than the conventional system.

We begin with a description of the new system, and then describe the simulation system. We conclude with results from the simulation.

\subsection{A hub and feeder public transport system}

The hub node is a central concept in the system. Hub nodes are carefully chosen to minimise the number of vehicle changes that passengers are required to make, by situating them at stops that are popular origins or destinations.

A high-frequency bus service links the hub nodes efficiently. Routes are designed so that travelling from one hub to another can usually be done without the need to change buses. We aim to have buses departing at around 10-minute intervals.

Taxis operate as shuttles between local bus stops and the hubs. The taxis operate as multi-hire vehicles, so passengers may share the vehicle, and may deviate on their route to pick up other passengers.

The methods for choosing hub nodes and designing routes is described in a companion paper Choosing Efficient Hubs and Routes in an Innovative Public Transport System.

A bus-stop to bus-stop service is offered, using the same bus stops as the existing system. Although taxis are used, it is not a door-to-door service.

A marked difference in this system is that all travel must be booked in advance. The booking may be made using a telephone call centre, a booking web site, a phone app, or a booth at one of the hub nodes.

Details of the fare system have not been finalised, but one possibility is that passengers pay when booking their journey. The normal bus fare covers all taxi and bus legs.

When the passenger books, they are provided with a 10 minute window in which they will be picked up (by taxi) from their origin stop.

A typical usage scenario would be as follows

- A passenger rings to book a journey. They are given a window during which they must be at the origin bus stop.

- During that window, a taxi picks them up, and takes them to the nearest hub (possibly deviating to pick up another passenger). For many passengers, this is the end of their journey.

- Otherwise, they wait on average 5 minutes to board a bus to take them to the hub nearest their destination.

- When they arrive, if this hub is not their destination there is a taxi waiting, displaying their name, to complete the journey.

An advantage of the system is that the number of taxis in the system responds to the level of demand: as demand increases, new taxis are brought on and then released again as demand eases. This alleviates much of the financial burden of traditional bus services which must run a fixed number of vehicles regardless of the number of passengers. Furthermore, it is our hope that by removing a large number of routes and instead providing much more direct shared-taxi journeys, our system will offer significantly reduced travel times for passengers.

\subsection{Case Study: Canberra, Australia}

In this paper we simulate our proposed system using real journey and demand data acquired from the existing bus system in Canberra, Australia. Canberra is a city of 350,000 people in a geographically dispersed area.

We were provided a data from four weekends comprising actual trips in the existing system, including timestamped origin and destination data. Using this data, we concatenate all linked trips by a given passenger in order to obtain full journeys, and hence the level of demand between all bus-stop pairs in the system. 
Canberra is an ideal place to test the proposed system, since the population density is very low, and hence difficult to service in the off-peak period with a conventional bus system. For example, on Sunday 03/06/2012, a total of 4,528 journeys were made across Canberra's 2,847 bus stops. However, most of these journeys involved only a handful of stops: nearly $70 \%$ of stops were never used, and more than $85 \%$ of them were used four times or less during the day. It seems, therefore, that there is is much wasted effort in passing these stops.

The current low-frequency service does not offer passengers the convenience they desire, but even this reduced service is quite expensive for the local government to run. We try to address both these problems with a redesign of how off-peak public transport will be offered.

\subsection{Previous Work}

The proposed system falls under the umbrella term "Demand-Responsive Transport" (DRT), which is a very active field of innovative systems for improving public transport. In their report, Enoch et al. (2004) investigate many case-studies involving conventional public transport and DRT. They find that these case-studies can be classified into main types of composite:

- Interchange DRT - refers to DRT vehicles being used in order to increase the capacity of an existing public transport network.

- Network DRT refers to DRT vehicles which improve traditional public transport by improving the service, or replacing uneconomic services. This typically occurs at times when demand is insufficient to warrant a conventional network or in places too sparsely populated for conventional services.

- Destination-specific DRT these are similar to Network DRTs, however they service specific destinations.

- Substitute DRT in this setting a DRT is used to replace all or most of a conventional public transport system. This could be due to the existing system not receiving sufficient demand, or not being commercially viable.

The proposed system resembles existing systems designed for disabled or mobility impaired passengers. These systems are surveyed in Enoch et al. (2004); Levine (1997). In particular, Aldaihani and Dessouky (2003) propose a system of demand responsive ride-share vehicles which connect to an existing fixed schedule-service. In this sense their solution can be viewed as a Network DRT since it aims to improve the traditional service to meet the needs of a user-base which is otherwise too small to be serviced by traditional public transport measures. However, all these system differ from the one proposed here in that they do not seek to completely replace the existing fixed-schedule service.

Destination-specific DRTs such as the Ipswich (UK) shared-taxi system reported in Balcombe et al. (1990) and the highly successful San Francisco Bay Area train feeder service discussed in Cervero et al. (1995) are generally many-to-one or one-to-many. For example the Ipswich system delivered passengers to locations around town, departing from the local train station only. Although the bay area network connects to several different train stations, it is still considered Destination specific.

Again the system proposed in this paper is different, being a many-to-many system.

\section{Simulation System}

We allow the passenger to specify their journey using either "depart after" or "arrive by" constraints. In the "depart after" scenario the passenger is given an itinerary which aims to deliver them to their destination at the earliest time possible. In the latest depart "arrive-by" scenario, on the other hand, the passenger is given an itinerary which aims to pick the passenger up at the latest possible time in order to arrive by their specified arrival time.

In order to test and cost the proposed system, the a simulator was developed which models several entities:

- Bus Stops $N$ are configured geographically as they are in Canberra

- Hub Stops $H \subset N$. A small subset of the aforementioned bus stops are designated to be hubs.

- Passengers enter with a desired origin, destination and travel times. The origin and destination may be any stops $i, j \in N$. system. 
- Buses run on a small set of predefined routes $R$, only stopping at the predefined hub stops $H$. These scheduled services run at a with a given target frequency.

- Taxis transport the passengers between the bus stops $N \backslash H$ and the hub stops $H$.

- Journeys are presented as a list of tuples $\left(i, t^{\text {start }}, t^{\text {stop }}, n^{\text {start }}, n^{\text {stop }}\right)$ whereby a passenger with id $i$ wishes to travel from $n^{\text {start }}$ to $n^{\text {stop }}$. The passenger books their journey at time $t^{\text {start }}$. If a non-zero $t^{s t o p}$ is specified, then an itinerary will be found which gets the passenger to their destination by time $t^{\text {stop }}$. Otherwise, the passenger will be given an itinerary which departs as soon as possible after $t^{\text {start }}$.

- Distance/Time Matrices $d(i, j)$ and $t(i, j)$ respectively specify the distance and time between stops $i, j \in N$. Both have zero diagonal.

Several parameters can be adjusted in the simulator. They are given here along with the setting used in the reported results.

- Transfer Time $t^{\text {transfer }}$ specifies the amount of time it takes for a passenger to transfer between taxibus, bus-taxi, bus-bus, and taxi-taxi. (2 mins)

- Bus Stop Wait Time $t^{\text {wait }}$ specifies the maximum time a passenger must wait for a taxi at a bus stop. (10 mins)

- Book-ahead Time $t^{b o o k-a h e a d}$ specifies the minimum time before desired departure that a passenger may book their trip. (10 mins)

- Taxi Stop Duration $t^{\text {taxi-stop }}$ specifies the time taken for a taxi to stop at a bus stop and for the passenger to board. (30 seconds)

- Maximum Taxi Deviation Multiplier $\lambda \geq 1$ specifies the amount by which a taxi may deviate from a direct trip in order to allow other pickups and set-downs along the way. That is, for a taxi trip which would take time $t$ to execute directly, we allow up to $\lambda t$ so that the taxi can perform other operations along the way. $(\lambda=1.5)$

\section{Taxi Dispatch}

We treat the problem of taxi dispatch in our system as a modified dial-a-ride problem (DARP) - see Berbeglia et al. (2010) for a recent survey of Dynamic Dial-a ride problems. Unlike many previous systems, the DARP we solve here features a very short book-ahead time - typically only ten minutes. Another atypical feature is that some requests in our system require bookings at both the source and destination of the trip. That is, for a passenger travelling from $A$ to $B$ through hubs $H_{A}$ and $H_{B}$ there are two linked DARPs to be solved one from $A$ to $H_{A}$, and one from $H_{B}$ to $B$, with the linking constraint that the desired pickup time at $H_{B}$ is determined by the bus departure time at $H_{A}$.

The pickup times of taxis are constrained by both the passenger's request, and the bus timetable. Unlike many systems which provide feeders to conventional public transport, the proposed system uses bus routes specifically designed for the taxi feeder system. This results in a higher frequency bus service which allows our DARP solver greater flexibility to meet the passenger specified constraints.

When a new booking is received, it is inserted into the schedule in the feasible position which results in least increase in cost using a simple insertion scheme. However, the whole DARP is periodically re-solved to improve cost. In this process, requests for some period in the future (approximately 5 minutes) are "locked in". All other requests are submitted to a general-purpose vehicle routing solver called Indigo (Kilby and Verden, 2011). The solver is allowed to run for one minute. At the end of that time, the best solution is returned. Any requests that have arrived during the solve are inserted in the new schedule using the usual insertion procedure. If they can be inserted successfully, the new schedule is adopted as the incumbent. At the moment this re-solve process is repeated every 10 (simulated) minutes.

\section{Simulation Informed Features}

One area where the simulation system helped guide the design of the system was in the are of use of direct taxis. Originally, the concept had been that passengers were always taken to their nearest hub. For example, 
bus stop $A$ may have closest hub $H$. If their destination bus $B$ stop also has closest hub $H$, they would catch a (possibly different taxi) from $H$ to $B$. However going direct from $A$ to $B$ will always be shorter (or at least no longer) than going via $H$ - for both passenger and taxi. We saw in the simulation that passengers would often go to the hub in a taxi, then immediately head off to their destination. We therefor allow direct routes when both source and destination have the same hub.

The same logic can apply even when the source and destination have different hubs. Let $A$ and $B$ have nearest hubs $H_{A}$ and $H_{B}$ respectively. If the distance $A$ to $B$ is less than distance $A$ to $H_{A}$ plus distance $B$ to $H_{B}$, then it is more efficient - for both taxi and passenger, to travel direct to destination, rather than via the hubs.

Through extensive simulation we determined that the best passenger travel time to cost tradeoff was achieved with relatively lower numbers of hubs compared with our initial expectations. We determined that, by having a smaller number of hubs, there was a higher amount of demand for each hub, thus enabling the taxis greater scope for trip optimisation.

Another design philosophy overturned using results from the simulator was around the bus frequency. Originally, the design goal had been to have a high frequency all day. However, looking at the results from the simulation, we saw that morning and evening the demand is very low. We were able to show that reducing frequency at these times did not result in unduly long travel times, but gave significant cost savings.

One area currently under investigation is the idea of "sub-hubs". In many cases, the simulation shows us that taxis drive along, or close, to the route of a bus. The question arises, should the taxi drop off their passengers at an intermediate point on the way to the hub, for the bus to pick up. We are currently investigating the effect on travel time and convenience that would result from such a change.

\section{Simulation Results}

In this section we report the results of the simulation of the proposed system.

We used bus costs estimated from publicly available data from the Canberra Budget papers. These estimates have yet to be confirmed by the transport authority or the bus company, and so must be treated as only approximate at the moment.

Costs for taxis are standard taxi company rates as reported on the Canberra Elite taxi company website.

The first question to be asked is, what is the appropriate number of hubs? We experimented with values up to 13 , but best results were obtained values around 3 . We report here the results for 2, 3, 4, and 5 hubs.

In Figure 1, we show the results for various numbers of hubs for a Saturday. This shows results for the current demand of approximately 7,000 journeys across the day. The best scenario for cost is 3 hubs, but best service is obtained with just 2 hubs. We estimate the cost of delivering the current fixed-schedule service to be around $\$ 140 \mathrm{~K}$ (i,e, $\$ 140,000$ ), so the 3-hubs solution represents a saving of nearly $25 \%$. The current average travel time is 28 minutes, so even the 3-hub solution reduces travel time by more than one third. For people who decide they want to leave immediately, there is an additional saving of 20 minutes on top of that in expected time before their journey can begin.

Figures for Sunday are similar, with an approximate expected saving of $20 \%$ for 3 hubs.

\begin{tabular}{|l||r|r|r|r|}
\hline & 2 Hubs & 3 Hubs & 4 Hubs & 5 Hubs \\
\hline Total Cost & $\$ 111 \mathrm{~K}$ & $\$ 107 \mathrm{~K}$ & $\$ 110 \mathrm{~K}$ & $\$ 131 \mathrm{~K}$ \\
Taxi Cost & $\$ 102 \mathrm{~K}$ & $\$ 92 \mathrm{~K}$ & $\$ 86 \mathrm{~K}$ & $\$ 80 \mathrm{~K}$ \\
Bus Cost & $\$ 9 \mathrm{~K}$ & $\$ 15 \mathrm{~K}$ & $\$ 24 \mathrm{~K}$ & $\$ 51 \mathrm{~K}$ \\
Ave Journey Time & $15: 35$ & $17: 30$ & $18: 15$ & $18: 20$ \\
Num Taxis & 123 & 116 & 111 & 107 \\
\hline
\end{tabular}

Table 1. Comparison of key statistics for various numbers of hubs for a typical Saturday

The number of legs in each journey is interesting. Table 2 shows the proportion of journeys with 1, 2 and 3 legs. It also shows how many of the journeys represent single-taxi journeys (i.e., this is a subset of the 1-leg journeys). 
P. Kilby and M. Robards, Simulation Of an Innovative Public Transport System

\begin{tabular}{|l||r|r|r|r|}
\hline & 2 Hubs & 3 Hubs & 4 Hubs & 5 Hubs \\
\hline 1 Leg & $82 \%$ & $64 \%$ & $63 \%$ & $61 \%$ \\
2 Legs & $12 \%$ & $26 \%$ & $27 \%$ & $28 \%$ \\
3 Legs & $6 \%$ & $10 \%$ & $10 \%$ & $11 \%$ \\
One Taxi & $78 \%$ & $54 \%$ & $49 \%$ & $44 \%$ \\
\hline
\end{tabular}

Table 2. The proportion of journeys with 1, 2 and 3 legs, and the proportion that are single-taxi journeys

The number of taxis in use is relatively high for the system. In Figure 1 we show the number of taxis in use over the day for a 3-hub solution. The demand peaks at around 125 at midday on Saturday. While this is large number, there are 360 taxis in the largest taxi fleet in Canberra, and weekend mornings are periods of low demand. We therefore do not expect to have difficulties finding enough taxis to support the system.

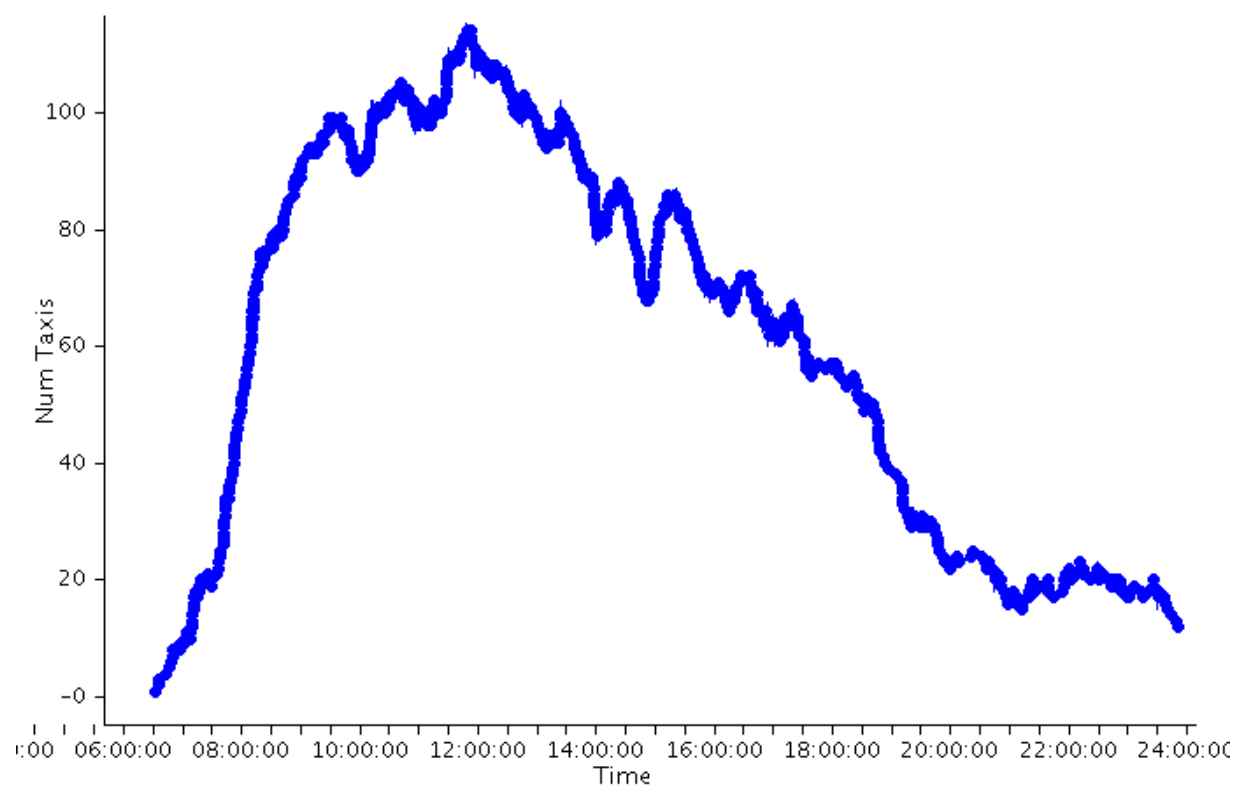

Figure 1. The number of taxis in use over a Saturday. The largest taxi company in Canberra has 360 vehicles

Finally, we demonstrate that bus occupancy justifies the use of the larger vehicles. Figure 2 shows the average occupancy of a bus over the main part of the day.

\section{Conclusions}

We have described a new public transport option designed for use in off-peak times. We have simulated its operation using current demand data from Canberra Australia. The initial results are encouraging. Travel times can be reduced by more than one third, while initial cost estimates indicate the system should cost less than the current fixed-schedule system. Simulation of the system has also lead to insights that have improved the design. Work on analysing other aspects of the system is continuing. A report for government is being prepared, using the results of the simulation.

\section{ACKNOWLEDGEMENT}

NICTA is funded by the Australian Government as represented by the Department of Broadband, Communications and the Digital Economy and the Australian Research Council through the ICT Centre of Excellence program 
Average passengers on a bus

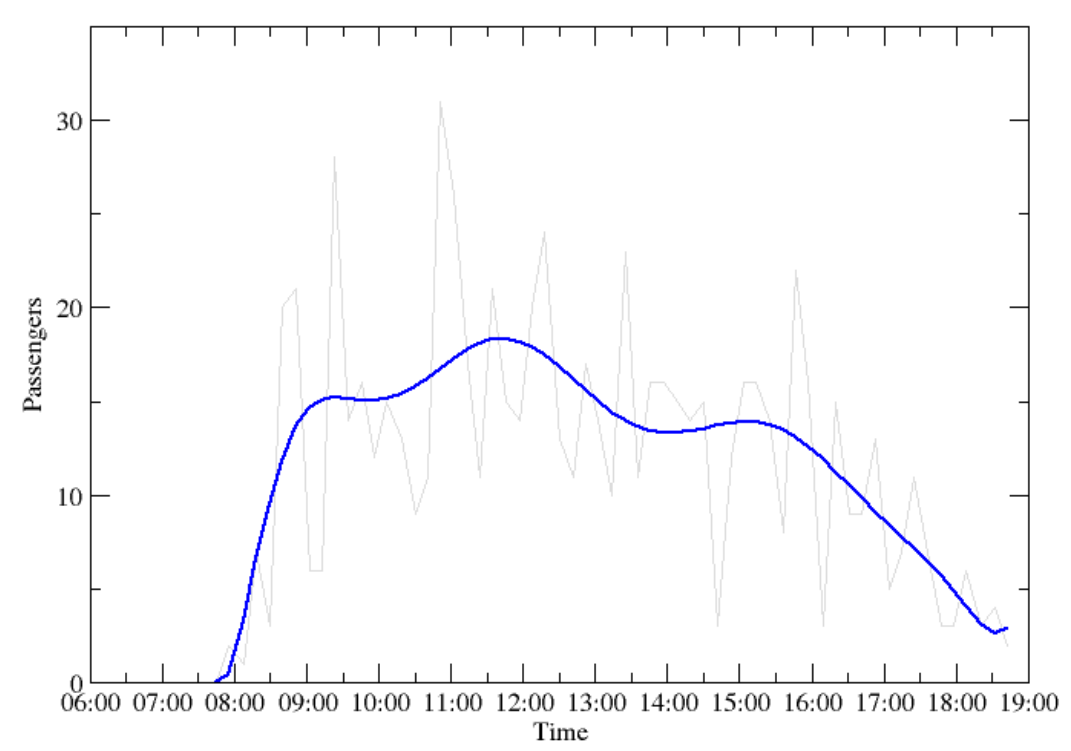

Figure 2. Average bus occupancy. The light line shows actual bus occupancy, while the darker line is a 10-minute running average

\section{REFERENCES}

Aldaihani, M. and M. M. Dessouky (2003). Hybrid scheduling methods for paratransit operations. Computers \& Industrial Engineering 45(1), 75-96.

Balcombe, R., D. Finch, D. Hollings, and H. Inwood (1990). Shared taxi schemes in britain: Lessons learned in ipswich. Technical report, Transport Research Laboratory (TRL).

Berbeglia, G., J.-F. Cordeau, and G. Laporte (2010). Dynamic pickup and delivery problems. European Journal Of Operational Research 202(1), 8-15.

Cervero, R., T. Kirk, D. Mount, and C. Reed (1995). Paratransit in the san francisco bay area: Providing feeder connections to rail. Working Paper qt9vp3z4sv, University of California Transportation Center.

Enoch, M., S. Potter, G. Parkhurst, and M. Smith (2004). Intermode: Innovations in demand responsive transport.

Kilby, P. and A. Verden (2011). Flexible routing combing constraint programming, large neighbourhood search, and feature-based insertion. In K. Schill, B. Scholz-Reiter, and L. Frommberger (Eds.), Proceedings 2nd Workshop on Artificial Intelligence and Logistics (AILOG-2011), pp. 43-48.

Levine, J. (1997). Ada and the demand for paratransit. Transportation Quarterly 51(1), 29-43. 